\title{
Nonlinear Decentralized Model Predictive Control for Unmanned Vehicles Moving in Formation
}

\author{
Alessandro Freddi ${ }^{1}$, Sauro Longhi ${ }^{2}$, Andrea Monteriù ${ }^{2}$ \\ ${ }^{1}$ Università degli Studi eCampus - Via Isimbardi 10, 22060 Novedrate (CO), Italy \\ e-mail: alessandro.freddi@uniecampus.it \\ ${ }^{2}$ Dipartimento di Ingegneria dell'Informazione \\ Università Politecnica delle Marche - Via Brecce Bianche - 60131 Ancona - Italy \\ e-mail:s.longhi@univpm.it,a.monteriu@univpm.it \\ crossref http://dx.doi.org/10.5755/j01.itc.44.1.7219
}

\begin{abstract}
Unmanned vehicles operating in formation may perform more complex tasks than vehicles working individually. In order to control a formation of unmanned vehicles, however, the following main issues must be faced: vehicle motion is usually described by nonlinear models, feasible control actions for each vehicle are constrained, collision between the members of the formation must be avoided while, at the same time, the computational efforts must be kept low due to limitations on the onboard hardware. To solve these problems, a nonlinear decentralized model predictive control algorithm is presented in this paper. The adopted model is based on the nonlinear kinematic equations describing the motion of a body with six degrees of freedom, where each vehicle shares information with its leader only by means of a wireless local area network. Saturation and collision-free constraints are included within the formulation of the optimization problem, while decentralization allows to distribute the computational efforts amongst all the vehicles of the formation. In order to show the effectiveness of the proposed approach, it has been applied to a formation of quadrotor vehicles. Simulation results prove that the approach presented in this paper is a valid way to solve the problem of controlling a formation of unmanned vehicles, granting at the same time the possibility to deal with constraints and nonlinearity while limiting the computational efforts through decentralization.
\end{abstract}

Keywords: autonomous vehicles; cooperative control; model predictive control; decentralized systems.

\section{Introduction}

In the last decades unmanned aerial, marine and ground vehicles have generated considerable attraction due to their strong autonomy and ability to perform difficult tasks in remote, uncertain or hazardous environments where human beings are unable to go.

The purposes of such vehicles are extremely various, ranging from scientific exploration, data collection and remote sensing, provision of commercial services, military reconnaissance and intelligence gathering. Recently, unmanned systems have become available and research is ongoing in a number of areas that will significantly advance the state of the art in unmanned vehicles technology. Moreover, designers have more freedom in the development of such vehicles, not having to account for the presence of a pilot and the associated life-support systems. This potentially results in cost and size savings, as well as increased operational capabilities, including fault diagnosis and fault tolerant supervision systems [1-5].
With an increasing availability of modern sensors and more effective communication channels, coordination of Unmanned Vehicles (UVs) has become feasible and widely studied in the recent years. Cooperative UVs can indeed provide significant benefit with respect to a single vehicle performing individually in a number of applications, such as remote sensing [6, 7], moving large objects [8] and a large number of them [9], exploration [10] and many others. In the literature three approaches are mainly studied for coordinating vehicles: behavioural methods, virtual structure techniques and leader-following approaches.

The basic idea of behavioural approaches is to assign desired behaviours to each agent, and to make the control action of each agent a weighted average of the control of the desired behaviours. Behaviour-based systems can thus integrate several goals, such as navigating to waypoints, avoiding hazards and keep formation at the same time [11-14]. The main advantage of behaviour-based systems is that they are decentralized, may be implemented with significantly 
less communication, explicit feedback is included through communication between neighbors and it is natural to derive control strategies when agents have multiple competing objectives. On the other side, however, they are difficult to analyse mathematically, it is hard to show that the formation has converged to the desired formation and the overall "group behaviour" may not be explicitly defined.

In the virtual structure approach, the entire formation is treated as a single entity: the desired motion is assigned to the virtual structure which traces out trajectories for each member of the formation to follow [1517]. In this approach, it is straightforward to assign a coordinated behavior for the group, and feedback to the structure is naturally defined. However, the necessity for the formation to act as a virtual structure limits the class of potential applications of this approach.

In leader-following approach, one vehicle is designated as the leader, while the rest of them is designated as followers. The idea is that followers track the position and orientation of the leader within a certain threshold. The hierarchy can be defined globally (whole formation) or locally (portions of formation), where in the latter case each vehicle takes another neighbor as a reference leader to determine its motion [18-23]. The strength of the leader-follower approach is that group behavior can be specified by setting the behavior of a single vehicle: the leader. Moreover it is a straightforward approach to implement and formation can be maintained even if the leader is perturbed by some disturbance. The main drawback is that there is no explicit feedback to the formation, that is no explicit feedback from the followers to the leader: if the follower is perturbed the formation may not be kept.

This paper presents an algorithm which can be adopted to maintain a formation of unmanned vehicles while they move along a desired trajectory, and it is based on leader-following architecture and Model Predictive Control (MPC). Leader-following has been chosen since it is based on a well defined mathematical model, the class of its potential applications is wide (differently from behavioural and virtual structure approaches) and the hierarchy can be locally defined: in this way the formation control problem can be formalized using local mathematical models. Model predictive control has then been chosen as control technique, since it already proved to be a reliable way to deal with decentralized formation control, is well adaptable to leader-following architectures and allows to minimize control complexity and easy reconfiguration of formation [24-28].

The first contribution of the proposed paper is that of proposing an algorithm which can be applied to a wide class of unmanned vehicles to simultaneously track a trajectory, keep a desired formation pattern and avoid obstacles. The algorithm can be applied to unmanned vehicles which are equipped with a velocity controller and whose control actions and dynamics are constrained (e.g. actuator saturations and physical limitation of the velocity and acceleration vectors). The second contribution is that the algorithm is based on a nonlinear model, which does not require to operate near one or more predefined working points, but can be used in a full range of operating conditions. The third contribution is that the formation control is faced in three dimensions, whereas many recent and advanced contributions in the literature only address the bidimensional problem [29-36], extending results previously obtained by the authors for the motion on the horizontal plane only [37-39]. Finally, the decentralization of the proposed control algorithm permits to decrease the computational load of the solution, which can be applied in many practical scenarios.

The paper is organized as follows. Section 2 describes the physical requirements which the vehicles must posses for the algorithm to be applied. In the same section, the kinematic model of each unmanned vehicle of the formation and the formation vector are derived. The proposed algorithm is detailed in Section 3. The proposed framework has been evaluated in a simulated scenario, in which formation control of quadrotor vehicles is addressed: Section 4 provides the results of the trial. Conclusions and future works are finally provided in Section 5.

\section{Mathematical Model of a UVs' Formation}

The vehicles of the formation considered in this paper possess a minimum set of requirements:

- Sensors: each vehicle must be equipped with an heading and an absolute localization sensor.

- Communication: vehicles must be connected to a WLAN to exchange sensor information and predicted control efforts; the required bandwidth is low since the algorithm requires only a limited amount of information (i.e. measurements and control effort predictions among each leader and its followers) to be transmitted.

- Low-level controller: each vehicle must posses an inner controller for the internal dynamics which can track a reference velocity vector.

These requirements are suitable for many practical situations where UVs are employed, and they are really common for many commercial UVs. When these requirements are satisfied, then it is possible to apply the Nonlinear Decentralized Model Predictive Control (ND-MPC) algorithm detailed in the following sections.

The vehicle formation is now modeled in order to predict the vehicles motion within a predictive horizon. These predictions allow to formulate a nonlinear optimization problem, whose solutions are the desired values of linear and angular velocities which must be tracked by each agent to maintain the desired formation and follow the desired trajectory.

\subsection{Kinematic Model of Each UV of the Formation}

Let consider a set of $N$ unmanned vehicles $\mathcal{V}^{i}(i=$ $1,2, \ldots, N)$. Each vehicle $\mathcal{V}^{i}$ moves autonomously at a 
fixed distance from another, following a leader vehicle $\mathcal{V}^{1}$ whose trajectory can be arbitrarily chosen (i.e., the trajectory of the virtual leader $\mathcal{V}^{0}$ ).

$N+1$ frames are used to study the formation motion (see Fig. 1): a frame integral with the earth $\{R\}(O, x, y, z)$, which is supposed to be inertial, and $N$ body-fixed frames $\left\{R_{B}^{i}\right\}\left(O_{B}^{i}, x_{B}^{i}, y_{B}^{i}, z_{B}^{i}\right)$, where $O_{B}^{i}$ is fixed to the center of mass of the $i$-th vehicle. $\left\{R_{B}^{i}\right\}$ is related to $\{R\}$ by a position vector $\xi^{i}(t)=$ $\left[\begin{array}{lll}x^{i}(t) & y^{i}(t) & z^{i}(t)\end{array}\right]^{T}$, describing the position of the center of gravity of vehicle $\mathcal{V}^{i}$ (i.e., $O_{B}^{i}$ ) with respect to $\{R\}$ and by a vector of three independent angles $\boldsymbol{\eta}^{i}(t)=\left[\begin{array}{lll}\phi^{i}(t) & \theta^{i}(t) & \psi^{i}(t)\end{array}\right]^{T}$, which represent the orientation of the vehicle (i.e., the orientation of the body-fixed frame $\left\{R_{B}^{i}\right\}$ ), with respect to the earth frame $\{R\}$, using the so-called yaw, pitch and roll notation (referred to as Euler angles, [40]).

In this way, $\xi^{i}(t)=\left[\begin{array}{lll}x^{i}(t) & y^{i}(t) & z^{i}(t)\end{array}\right]^{T}$ and $\boldsymbol{\eta}^{i}(t)=\left[\begin{array}{lll}\phi^{i}(t) & \theta^{i}(t) & \psi^{i}(t)\end{array}\right]^{T}$ fully describe, respectively, the translational and the rotational movement of the $i$-th vehicle with respect to the earth frame $\{R\}$.

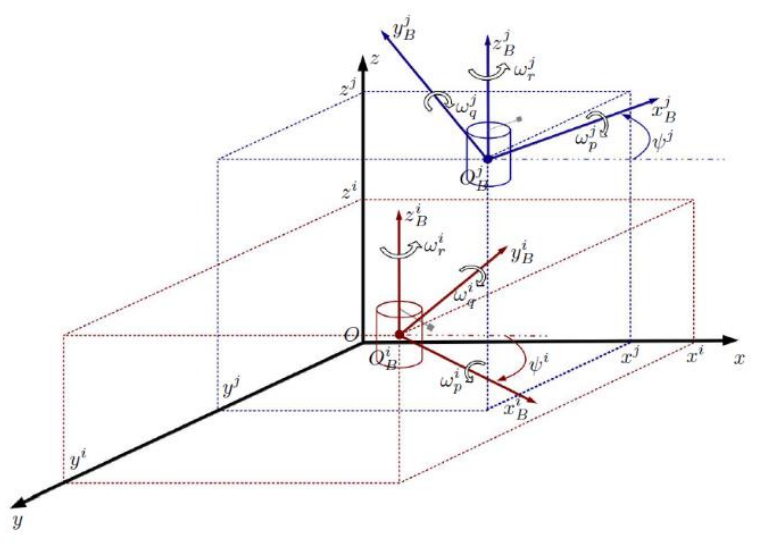

Figure 1. The reference frames adopted: each agent as a solidal body frame whose origin is in the center of gravity

Let define the rotation matrix which maps the linear velocity of $\mathcal{V}^{i}$ from the $i$-th body frame into the earth frame as

$$
\mathbf{R}\left(\phi^{i}(t), \theta^{i}(t), \psi^{i}(t)\right) \triangleq\left[\begin{array}{ccc}
C_{\theta^{i}} C_{\psi^{i}} & C_{\psi^{i}} S_{\theta^{i}} S_{\phi^{i}}-C_{\phi^{i}} S_{\psi^{i}} & C_{\phi^{i}} C_{\psi^{i}} S_{\theta^{i}}+S_{\phi^{i}} S_{\psi^{i}} \\
C_{\theta^{i}} S_{\psi^{i}} & S_{\theta^{i}} S_{\phi^{i}} S_{\psi^{i}}+C_{\phi^{i}} C_{\psi^{i}} & C_{\phi^{i}} S_{\theta^{i}} S_{\psi^{i}}-C_{\psi^{i}} S_{\phi^{i}} \\
-S_{\theta^{i}} & C_{\theta^{i}} S_{\phi^{i}} & C_{\theta^{i}} C_{\phi^{i}}
\end{array}\right]
$$

where $S_{(.)}$and $C_{(.)}$represent $\sin ($.$) and \cos ($.$) ,$ respectively.

Moreover, let define the matrix which maps the angular velocity of $\mathcal{V}^{i}$ read in the $i$-th body frame into the rate of change of the Euler angles as

$$
\mathbf{W}\left(\phi^{i}(t), \theta^{i}(t)\right) \triangleq\left[\begin{array}{ccc}
1 & S_{\phi^{i}} T_{\theta^{i}} & C_{\phi^{i}} T_{\theta^{i}} \\
0 & C_{\phi^{i}} & -S_{\phi^{i}} \\
0 & S_{\phi^{i}} / C_{\phi^{i}} & C_{\phi^{i}} / C_{\theta^{i}}
\end{array}\right](2)
$$

where $T_{(.)}$represents tan (.). Denoting the linear velocity vector of the $i$-th vehicle along the axes of the $i$-th body frame as $\mathbf{v}^{i}(t)=\left[\begin{array}{lll}v_{x}^{i}(t) & v_{y}^{i}(t) & v_{z}^{i}(t)\end{array}\right]^{T}$, and the angular velocity vector of the $i$-th vehicle around the axes of the $i$-th body frame as $\boldsymbol{\omega}^{i}(t)=$ $\left[\omega_{p}^{i}(t) \omega_{q}^{i}(t) \omega_{r}^{i}(t)\right]^{T}$ (as described in Fig. 1), the kinematic model for the vehicle $\mathcal{V}^{i}$ can be described by

$$
\begin{aligned}
& \dot{\boldsymbol{\xi}}^{i}(t)=\mathbf{R}\left(\phi^{i}(t), \theta^{i}(t), \psi^{i}(t), \mathbf{v}^{i}(t)\right) \\
& \dot{\boldsymbol{\eta}}^{i}(t)=\mathbf{W}\left(\phi^{i}(t), \theta^{i}(t), \boldsymbol{\omega}^{i}(t)\right) .
\end{aligned}
$$

In particular, defining the absolute configuration vector (i.e., referred to the earth frame) for the $i$-th vehicle as

$$
\mathbf{q}^{i}(t) \triangleq\left[\begin{array}{llll}
x^{i}(t) & y^{i}(t) & z^{i}(t) & \psi^{i}(t)
\end{array}\right]^{T}
$$

and assuming that the roll $\left(\phi^{i}(t)\right)$ and pitch $\left(\theta^{i}(t)\right)$ angles of vehicle $\mathcal{V}^{i}$ are both close to zero (i.e., stabilized), the time-continuous kinematic model for the $i$-th vehicle can be derived from (3) as

$$
\dot{\mathbf{q}}^{i}(t)=\mathbf{T}^{-1}\left(\psi^{i}(t)\right) \mathbf{u}^{i}(t)
$$

$$
\begin{aligned}
& \mathbf{T}^{-1}\left(\psi^{i}(t)\right) \triangleq\left[\begin{array}{cccc}
C_{\psi^{i}} & -S_{\psi^{i}} & 0 & 0 \\
S_{\psi^{i}} & C_{\psi^{i}} & 0 & 0 \\
0 & 0 & 1 & 0 \\
0 & 0 & 0 & 1
\end{array}\right] \\
& \mathbf{u}^{i}(t) \triangleq\left[\begin{array}{llll}
v_{x}^{i}(t) & v_{y}^{i}(t) & v_{z}^{i}(t) & \omega_{r}^{i}(t)
\end{array}\right]^{T} .
\end{aligned}
$$

From a physical point of view, the constraints on roll and pitch angles imply that the linear dynamics, together with the rotational dynamics around the vertical axis, can be decoupled from the dynamics of roll and pitch. This is valid for ground vehicles, usually valid for marine vehicles and also valid for aerial vehicles in conservative flight conditions [41].

\subsection{Formation Vector Model}

Let assume to sample the continuous-time variables with sampling interval $T_{S}$ and let define the sampled input vector of each vehicle $\mathcal{V}^{i}$ as

$$
\mathbf{u}_{k}^{i} \triangleq\left[\begin{array}{llll}
v_{x_{k}}^{i} & v_{y_{k}}^{i} & v_{z_{k}}^{i} & \omega_{r_{k}}^{i}
\end{array}\right]^{T}, i=1,2, \ldots, N
$$

where, for $k \in \mathbb{N}$,

$$
\begin{aligned}
& \mathbf{u}_{k}^{i} \triangleq T_{s} \mathbf{u}^{i}\left(k T_{s}\right) \\
& v_{x_{k}}^{i} \triangleq T_{s} v_{x}^{i}\left(k T_{s}\right) \\
& v_{y_{k}}^{i} \triangleq T_{s} v_{y}^{i}\left(k T_{s}\right) \\
& v_{z_{k}}^{i} \triangleq T_{s} v_{z}^{i}\left(k T_{s}\right) \\
& \omega_{r_{k}}^{i} \triangleq T_{s} \omega_{r}^{i}\left(k T_{s}\right) .
\end{aligned}
$$

Eqs. (7) represent finite movements within each sampling interval $T_{s}$. These movements can also be 
seen as velocities normalized w.r.t. the sampling interval $T_{s}$ and, in the following, they will be referred to as velocities.

Let define the discretized absolute configuration vector for the $i$-th vehicle as

$$
\mathbf{q}_{k}^{i} \triangleq\left[\begin{array}{llll}
x_{k}^{i} & y_{k}^{i} & z_{k}^{i} & \psi_{k}^{i}
\end{array}\right]^{T}
$$

where, for $k \in \mathbb{N}$,

$$
\begin{aligned}
& \mathbf{q}_{k}^{i} \triangleq \mathbf{q}^{i}\left(k T_{s}\right) \\
& x_{k}^{i} \triangleq x^{i}\left(k T_{s}\right) \\
& y_{k}^{i} \triangleq y^{i}\left(k T_{s}\right) \\
& z_{k}^{i} \triangleq z^{i}\left(k T_{s}\right) \\
& \psi_{k}^{i} \triangleq \psi^{i}\left(k T_{s}\right) .
\end{aligned}
$$

Due to physical limits, the velocities of each vehicle are constrained and their limits depend on the lower level controller and on the dynamic behavior of each vehicle. Fixed constraints are assumed in the following without loss of generality

$$
\underline{\vec{u}} \preccurlyeq \vec{u}_{k}^{i} \preccurlyeq \overline{\vec{u}}
$$

and

$$
\bmod \left(\Delta \vec{u}_{k}^{i}\right) \preccurlyeq \bmod (\Delta \vec{u})
$$

with

$$
\begin{aligned}
& \underline{\vec{u}}=\left[\begin{array}{llll}
\underline{v}_{x} & \underline{v}_{y} & \underline{v}_{z} & \underline{\omega}_{r}
\end{array}\right]^{T} \\
& \underline{\vec{u}}=\left[\begin{array}{llll}
\bar{v}_{x} & \bar{v}_{y} & \bar{v}_{z} & \bar{\omega}_{r}
\end{array}\right]^{T} \\
& \Delta \vec{u}_{k}^{i}=\left[\begin{array}{llll}
\Delta v_{x_{k}}^{i} & \Delta v_{y_{k}}^{i} & \Delta v_{z_{k}}^{i} & \Delta \omega_{r_{k}}^{i}
\end{array}\right]^{T} \\
& \Delta \vec{u}=\left[\begin{array}{llll}
\Delta v_{x} & \Delta v_{y} & \Delta v_{z} & \Delta \omega_{r}
\end{array}\right]^{T}
\end{aligned}
$$

where $\underline{v}_{x}, \underline{v}_{y}, \underline{v}_{z}$ and $\bar{v}_{x}, \bar{v}_{y}, \bar{v}_{z}$ are the constant linear velocity bounds, $\underline{\omega}_{r}$ and $\bar{\omega}_{r}$ are the constant angular velocity bounds, while linear and angular velocities variations are limited by $\Delta v_{x}, \Delta v_{y}, \Delta v_{z}$ and $\Delta \omega_{r}$, respectively. In Eq. (10), $\vec{f} \preccurlyeq \vec{g}$ denotes that for each $j$-th element of the two vectors $\vec{f}$ and $\vec{g}, f_{j} \leq g_{j}$ holds, and in Eq. $(11), \bmod (\vec{f})=\left[\left|f_{1}\right|\left|f_{2}\right| \ldots\left|f_{j}\right|\right]^{T}$ is the modulus of each element $f_{j}$ of a vector $\vec{f}$. In detail, denoting with $\Delta v_{()_{k}}^{i}=v_{(.)_{k}}^{i}-v_{()_{k-1}}^{i}$ the change at time $k$ of a scalar $v_{(.)}$, the physical limits can be resumed as follows

$$
\begin{array}{ll}
\underline{v}_{x} \leq v_{x_{k}}^{i} \leq \bar{v}_{x}, & \left|\Delta v_{x_{k}}^{i}\right| \leq \Delta v_{x} \\
\underline{v}_{y} \leq v_{y_{k}}^{i} \leq \bar{v}_{y}, & \left|\Delta v_{y_{k}}^{i}\right| \leq \Delta v_{y} \\
\underline{v}_{z} \leq v_{z_{k}}^{i} \leq \bar{v}_{z}, & \left|\Delta v_{z_{k}}^{i}\right| \leq \Delta v_{z} \\
\underline{\omega}_{r} \leq \omega_{r_{k}}^{i} \leq \bar{\omega}_{r}, & \left|\Delta \omega_{r_{k}}^{i}\right| \leq \Delta \omega_{r} .
\end{array}
$$

The time continuous Eqs. (5) can now be discretized into

$$
\mathbf{q}_{k+1}^{i}=\mathbf{q}_{k}^{i}+\mathbf{T}^{-1}\left(\psi_{k}^{i}\right) \mathbf{u}_{k}^{i} .
$$

Defining the displacement of vehicle $\mathcal{V}^{j}$ referred to the frame fixed to vehicle $\mathcal{V}^{i}$ as

$$
\mathbf{d}_{k}^{j i} \triangleq\left[\begin{array}{llll}
d_{x_{k}}^{j i} & d_{y_{k}}^{j i} & d_{z_{k}}^{j i} & d_{\psi_{k}}^{j i}
\end{array}\right]^{T}
$$

where $d_{(.)_{k}}^{j i}$ represents the linear and angular distances among vehicles, the absolute configuration vector can be expressed as

$$
\mathbf{d}_{k}^{j i}=\mathbf{T}\left(\psi_{k}^{i}\right)\left(\mathbf{q}_{k}^{j}-\mathbf{q}_{k}^{i}\right)
$$

which leads to the following discrete-time formation vector model

$$
\mathbf{d}_{k+1}^{j i}=\mathbf{A}_{k}^{i} \mathbf{d}_{k}^{j i}+\mathbf{B}_{k}^{i} \mathbf{u}_{k}^{i}+\mathbf{E}_{k}^{j i} \mathbf{u}_{k}^{j}
$$

where

$$
\begin{aligned}
& \mathbf{A}_{k}^{i} \triangleq \mathbf{T}\left(\omega_{r_{k}}^{i}\right) \\
& \mathbf{B}_{k}^{i} \triangleq-\mathbf{T}\left(\omega_{r_{k}}^{i}\right) \\
& \mathbf{E}_{k}^{j i} \triangleq \mathbf{T}\left(\omega_{r_{k}}^{i}\right) \mathbf{T}^{-1}\left(d_{\psi_{k}}^{j i}\right) .
\end{aligned}
$$

\section{Formation Control}

The formation control algorithm proposed in this paper is based upon the following two sets of assumptions.

\section{Assumptions Set 1.}

(a) The reference trajectory $\mathcal{T}^{*}$ is generated by a virtual reference vehicle $\mathcal{V}^{0}$ which moves according to the considered model.

(b) Each vehicle $\mathcal{V}^{i}$ follows one and only one leader $\mathcal{V}^{j}, j \neq i ; \mathcal{V}^{1}$ follows virtual vehicle $\mathcal{V}^{0}$ which exactly tracks the reference trajectory $\mathcal{T}^{*}$.

In the proposed control algorithm, each vehicle $\mathcal{V}^{i}$ is equipped with an independent control agent $\mathcal{A}^{i}$ whose tasks are to collect both local and remote information and iteratively perform a nonlinear optimization for computing the local control action. Each vehicle $v^{i}$ must keep the reference formation pattern from its leader $\mathcal{V}^{j}$, i.e., $\tilde{\mathbf{d}}^{j i}=\left[\begin{array}{llllll}\tilde{d}_{x}^{j i} & \tilde{d}_{y}^{j i} & \tilde{d}_{z}^{j i} & \tilde{d}_{\psi}^{j i}\end{array}\right]^{T} . \quad$ The set of all displacements defines the formation. In the implementation of the proposed formation control law, the following set of assumptions is made as well.

\section{Assumptions Set 2.}

(a) Each control agent $\mathcal{A}^{i}$ communicates with its leader using a WLAN only once within a sampling interval.

(b) The communication network introduces a delay $\tau=1$

(c) The agents are synchronous.

(d) Each control agent knows the absolute configurations of its leader.

Note that condition $(d)$ can be achieved through direct measurements, however when one or more absolute measurements are missing, an estimation provided by proper sensor fusion algorithms can be used as well [42]. 
The formation control problem is then decomposed into an inner-loop dynamic task, which consists of making the vehicle's velocities track a set of references, and an outer-loop kinematic task, which assigns the reference velocities to be tracked for the desired trajectory.

\subsection{Inner-Loop Dynamics Controller}

For each vehicle $\mathcal{V}^{i}$, the low level controller is assumed to drive the actuators in order to track the desired velocities $\tilde{v}_{x}^{i}, \tilde{v}_{y}^{i}, \tilde{v}_{z}^{i}, \widetilde{\omega}_{r}^{i}$. Note that this is possible if the inner control loop acts much faster than the outer control loop. With these assumptions, the considered high level control problem becomes a path planning problem for the low level controller. The high level controller should define the optimal speeds $\tilde{v}_{x}^{i}, \tilde{v}_{y}^{i}, \tilde{v}_{z}^{i}, \widetilde{\omega}_{r}^{i}$ that allow to keep the desired formation with the minimum possible efforts. The formation input vector $\mathbf{u}_{k}^{i}=\left[\begin{array}{llll}v_{x_{k}}^{i} & v_{y_{k}}^{i} & v_{z_{k}}^{i} & \omega_{r_{k}}^{i}\end{array}\right]^{T}$ is the reference vector for the low level controller. No other communication is needed between the two control loops, since the MPC requires position feedback only, as described below.

\subsection{Nonlinear Decentralized MPC Formulation}

The following scalar is considered here as a measure of the performance for control agent $\mathcal{A}^{i}$ :

$$
\begin{aligned}
& \left\langle\mathbf{d}_{k}^{j i}-\tilde{\mathbf{d}}^{j i}\right\rangle^{2} \triangleq \rho_{x}\left(d_{x_{k}}^{j i}-\tilde{d}_{x}^{j i}\right)^{2}+ \\
& \rho_{y}\left(d_{y_{k}}^{j i}-\tilde{d}_{y}^{j i}\right)^{2}+\rho_{z}\left(d_{z_{k}}^{j i}-\tilde{d}_{z}^{j i}\right)^{2}+ \\
& \rho_{\psi}\left[\sin \left(\frac{d_{\psi_{k}}^{j i}-\tilde{d}_{\psi}^{j i}}{2}\right)\right]
\end{aligned}
$$

where $\tilde{\mathbf{d}}^{j i}$ is the constant desired displacement, and $\rho_{x}$, $\rho_{y}, \rho_{z}, \rho_{\psi}$ are arbitrary weights. The cost function to be minimized is

$$
\begin{aligned}
& J_{k}^{i}=\sum_{h=1}^{p}\left[\left\langle\tilde{\mathbf{d}}_{k+h \mid k}^{j i}-\tilde{\mathbf{d}}^{j i}\right\rangle^{2}+\mu\left\|\widehat{\mathbf{u}}_{k+h-1 \mid k}^{i}\right\|^{2}+\right. \\
& \left.\sigma\left\|\Delta \widehat{\mathbf{u}}_{k+h-1 \mid k}^{i}\right\|^{2}\right]+\lambda \sum_{h=1}^{p-1} \| \widehat{\mathbf{u}}_{k+h-1 \mid k}^{i}- \\
& \widehat{\mathbf{u}}_{k+h-1 \mid k-1}^{i} \|^{2}
\end{aligned}
$$

where

- $\mu$ is the weight which penalizes the control efforts at time $k$;

- $\sigma$ is the weight which penalizes large variation of the control efforts;

- $\lambda$ is the weight which penalizes the variation of the control efforts between two successive predictions;

- $\quad p$ is the prediction horizon;

- $\widehat{\mathbf{u}}_{k+h \mid k}^{i}$ is the $h$-ahead prediction of the $i$-th vehicle control effort at time $k$.

The parameters are chosen as trade-off between MPC convergence speed, control efforts magnitude and computational complexity.
In order to avoid possible contacts between vehicles, a collision-free constraint is finally added to the set of velocities constraints (13)

$$
\begin{aligned}
& \left\|\mathbf{M}\left(\mathbf{A}_{k}^{i} \mathbf{d}_{k}^{l i}+\mathbf{B}_{k}^{i} \mathbf{u}_{k}^{i}\right)\right\| \geq \underline{d}+\sqrt{2} \bar{v} \\
& (l=1, \ldots, N, l \neq i)
\end{aligned}
$$

where $\underline{d}$ is the safe constant distance,

$$
\bar{v}=\max \left(\left|\underline{v}_{x}\right|,\left|\bar{v}_{x}\right|\right)+\max \left(\left|\underline{v}_{y}\right|,\left|\bar{v}_{y}\right|\right)
$$

and

$$
\mathrm{M}=\left[\begin{array}{llll}
1 & 0 & 0 & 0 \\
0 & 1 & 0 & 0
\end{array}\right]
$$

This constraint implies that the distance between two vehicles can not be less than a safe distance which is a control design parameter, and thus it can be arbitrarily fixed. This distance is typically chosen to be equal to twice the maximum dimension of the vehicle plus a safe threshold.

In order to guarantee that a vehicle remains within a limited region of the formation, the optimization problem is modified according to the approach proposed in [43]. In detail, a new auxiliary vector $\mathbf{a}_{k}^{i}$ is introduced, which has the same dimension of the state vector $\mathbf{d}_{k}^{j i}$, and whose dynamics is described by $\mathbf{a}_{k+1}^{i}=$ $\mathbf{S}^{i} \mathbf{a}_{k}^{i}$. The dynamic matrix $\mathbf{S}^{i}$ is chosen to be diagonal and its eigenvalues $s_{\xi}^{i}$ such that the linear dynamics of the new auxiliary vector is asymptotically stable. Using the new auxiliary vector, the error between the system states (actual distances between vehicles) and the desired states (desired distances between vehicles) is now enforced to be contracted with respect to the auxiliary variables by the inequality constraint

$$
\begin{aligned}
& \tilde{J}_{k}^{i}=\sum_{h=1}^{p}\left[\left\langle\tilde{\mathbf{d}}_{k+h \mid k}^{j i}-\tilde{\mathbf{d}}^{j i}\right\rangle^{2}+v\left\|\hat{\mathbf{a}}_{k+h-1 \mid h}^{i}\right\|^{2}+\right. \\
& \left.\mu\left\|\widehat{\mathbf{u}}_{k+h-1 \mid k}^{i}\right\|^{2}+\rho\left\|\Delta \widehat{\mathbf{u}}_{k+h-1 \mid k}^{i}\right\|^{2}\right]+ \\
& \lambda \sum_{h=1}^{p-1}\left\|\widehat{\mathbf{u}}_{k+h-1 \mid k}^{i}-\widehat{\mathbf{u}}_{k+h-1 \mid k-1}^{i}\right\|^{2}
\end{aligned}
$$

where $v$ is again a weight which can be arbitrary chosen.

The ND-MPC problem for the control agent $\mathcal{A}^{i}$ of each vehicle $\mathcal{V}^{i}$ can finally be expressed as

$$
\begin{aligned}
& \min _{\mathbf{d}_{k}^{j i}-\tilde{\mathbf{d}}^{j i}, \mathbf{a}_{k^{i}, \vec{S}^{i}, \mathbf{u}_{k}^{i}}} \tilde{J}_{k}^{i} \\
& \text { s.t. } \quad \text { a) } \mathbf{d}_{k+1}^{j i}=\mathbf{A}_{k}^{i} \mathbf{d}_{k}^{j i}+\mathbf{B}_{k}^{i} \mathbf{u}_{k}^{i}+\mathbf{E}_{k}^{j i} \mathbf{u}_{k}^{j} \\
& \text { b) } \mathbf{a}_{k+1}^{i}=\mathbf{S}^{i} \mathbf{a}_{k}^{i} \\
& \text { c) } \mathbf{S}^{i}=\operatorname{diag}\left(s_{\xi}^{i}\right),\left|s_{\xi}^{i}\right|<1 \\
& \text { d) }\left\|\mathbf{d}_{k}^{j i}-\tilde{\mathbf{d}}_{k}^{j i}\right\| \leq\left\|\mathbf{a}_{k}^{i}\right\|+\underline{d} \\
& \text { e) }\left\|\mathbf{M}\left(\mathbf{A}_{k}^{i} \mathbf{d}_{k}^{l i}+\mathbf{B}_{k}^{i} \mathbf{u}_{k}^{i}\right)\right\| \geq \underline{d}+\sqrt{2} \bar{v} \\
& \text { f) } \underline{\mathbf{u}} \preccurlyeq \mathbf{u}_{k}^{i} \preccurlyeq \overline{\mathbf{u}} \\
& \text { g) } \bmod \left(\Delta \vec{u}_{k}^{i}\right) \preccurlyeq \bmod (\Delta \vec{u}) .
\end{aligned}
$$

This nonlinear constrained optimization problem is iteratively set-up and solved at each sample time, 
allowing to compute the control efforts $\mathbf{u}_{k}^{i}$ for each $\mathcal{V}^{i}$ of the formation.

Several methods can be adopted to solve this nonlinear, constrained optimization problem, such as interior-point, trust-region-reflective, sqp and activeset algorithms [44], particle swarm based algorithms [45] and others.

\section{A case study: simulation results for a formation of quadrotors}

The developed strategy has been tested in a simulated scenario, created using MATLAB ${ }^{\circledR}$ to control a formation composed by $N=5$ quadrotor vehicles, namely $\mathcal{V}^{1}, \ldots, \mathcal{V}^{5}$, where $\mathcal{V}^{1}$ is the main leader. A quadrotor consists of four DC motors on which propellers are fixed. These motors are arranged to the extremities of a X-shaped frame, where all the arms make an angle of 90 degrees with one another. The speed of rotation of the motors (i.e., the lift force associated to the propeller attached to that motor) can be individually changed, thus modifying the attitude of the vehicle allowing the quadrotor to translate into the space. Quadrotor vehicles have been chosen since they are often used in literature as UAVs, due to their high manoeuvrability, simple modelling and low maintenance costs [46].

The starting formation and the objective one, are described in Fig. 2.
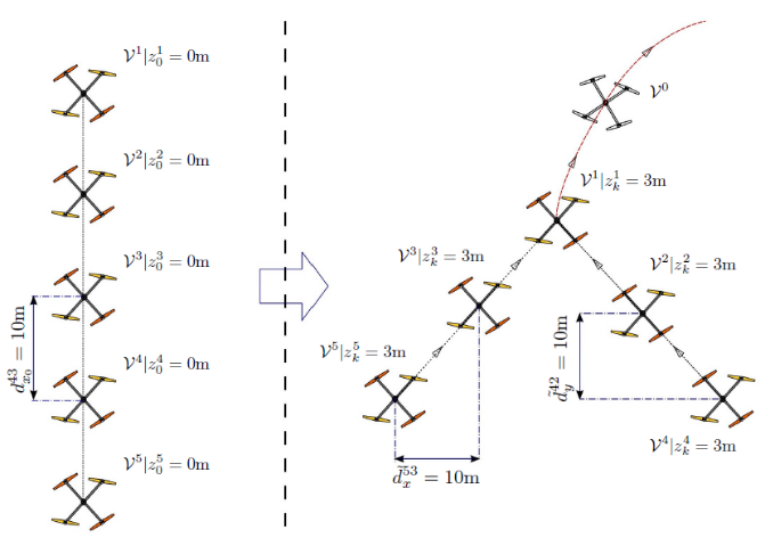

Figure 2. The considered leader-follower formation: the ND-MPC has the task to maintain a "V" formation of five vehicles, flying on the same plane at a fixed distance, while following the main leader

The physical constraints used in simulation are:

$$
\begin{aligned}
-2.5 \mathrm{~ms}^{-1} & \leq v_{x_{k}}^{i} \leq 2.5 \mathrm{~ms}^{-1} \\
-2.5 \mathrm{~ms}^{-1} & \leq v_{y_{k}}^{i} \leq 2.5 \mathrm{~ms}^{-1} \\
-2.5 \mathrm{~ms}^{-1} & \leq v_{Z_{k}}^{i} \leq 2.5 \mathrm{~ms}^{-1} \\
-0.075 \mathrm{rad} \mathrm{s}^{-1} & \leq \omega_{r_{k}}^{i} \leq 0.075 \mathrm{rad} \mathrm{s}^{-1}
\end{aligned}
$$

and

$$
\begin{aligned}
& \left|\Delta v_{x_{k}}^{i}\right| \leq 0.25 \mathrm{~ms}^{-2} \\
& \left|\Delta v_{y_{k}}^{i}\right| \leq 0.25 \mathrm{~ms}^{-2} \\
& \left|\Delta v_{z_{k}}^{i}\right| \leq 0.25 \mathrm{~ms}^{-2}
\end{aligned}
$$

$$
\left|\Delta \omega_{r_{k}}^{i}\right| \leq 0.005 \operatorname{rad~s}^{-2}
$$

with $i=1, \ldots, 5$.

The parameters used in simulation are:

- minimum safe distance $\underline{d}=0.5 \mathrm{~m}$,

- cost function weights: $\rho_{x}=\rho_{y}=\rho_{z}=10, \rho_{\psi}=$ $200, v=0.5, \mu=0.5, \sigma=1$ and $\lambda=0.4$,

- prediction horizon $p=3$,

- safe distance $\underline{d}$ equal to $0.75 \mathrm{~m}$

The total number of simulation steps is $K=350$, the chosen trajectory has a curvilinear behaviour and the optimization method chosen is based on an activeset algorithm.

As it can be seen in Fig. 3 the main leader $\mathcal{V}^{i}$ follows the virtual leader $\mathcal{V}^{0}$ while the followers quickly assume the desired formation, reaching the same height of the main leader and keeping the desired distances.

The transient phase for this kind of configuration is short. A similar result can be achieved changing the reference trajectory and the formation pattern. During this transient time the vehicle positions differ from the desired position, however at steady state all the distances between leaders and followers converge to the desired values, as it can be seen in Fig. 4 which shows the $d_{x_{k}}^{j i}, d_{y_{k}}^{j i}, d_{z_{k}}^{j i}$ and $d_{\psi_{k}}^{j i}$ variables representing lateral, longitudinal, vertical and angular relative distances between vehicle $\mathcal{V}^{j}$ and vehicle $\mathcal{V}^{i}$ at time $k$, namely the components of the displacement vector $\mathbf{d}_{k}^{j i}$. The dotted black lines represent the desired displacement values, while the colored solid lines represent the real displacement values.

\section{Conclusions}

Unmanned vehicles operating in formation may perform more complex tasks and better than single vehicles working individually. The formation control problem, however, is difficult to solve for this kind of vehicles since they are by nonlinear models and the control actions are constrained. Moreover the computational capabilities available to unmanned vehicles is usually limited, thus decentralized solutions are to be preferred to the centralized ones.

To solve the problem of formation control subject to trajectory following, a nonlinear decentralized model predictive control algorithm is developed in this paper, adopting leader-following: each vehicle moves keeping a fixed distance from its leader, where the main leader follows a desired trajectory. A nonlinear optimization problem is formulated within a certain prediction horizon, whose solutions are the desired values of linear and angular velocities which must be tracked by each vehicle to maintain the desired formation and follow the desired trajectory. In this way whenever the desired velocities can be correctly set by a low-level controller, the high-level formation control is achievable taking into account model constraints, actuation constraints and collision-free constraints. 


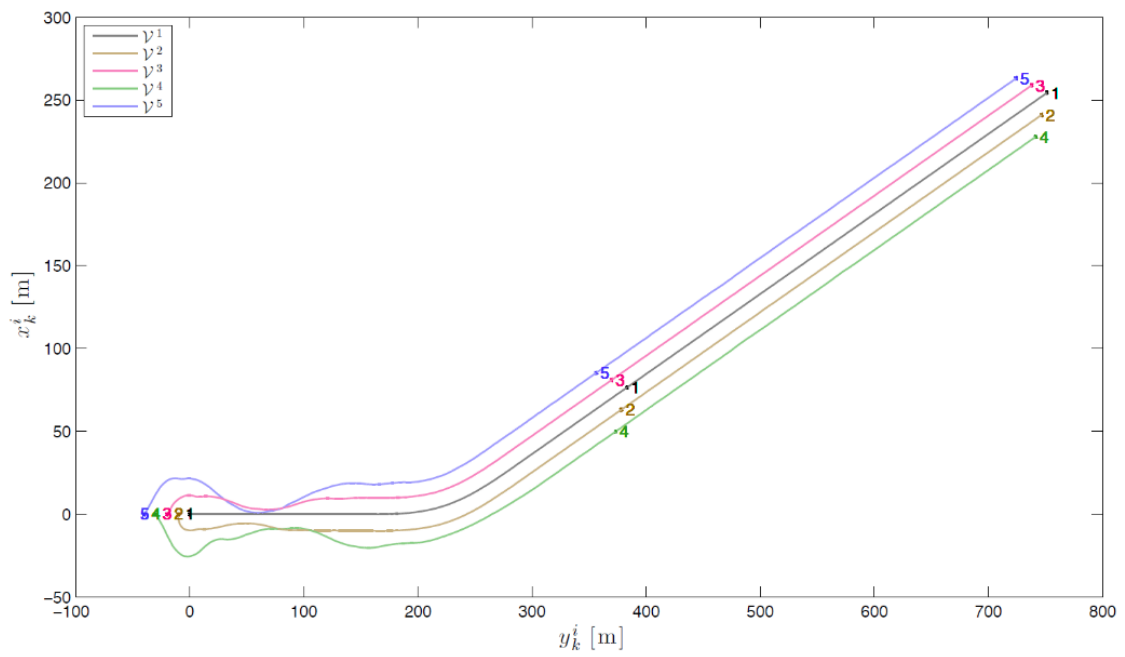

(a) Quadrotors trajectories projected into the Y-X plane

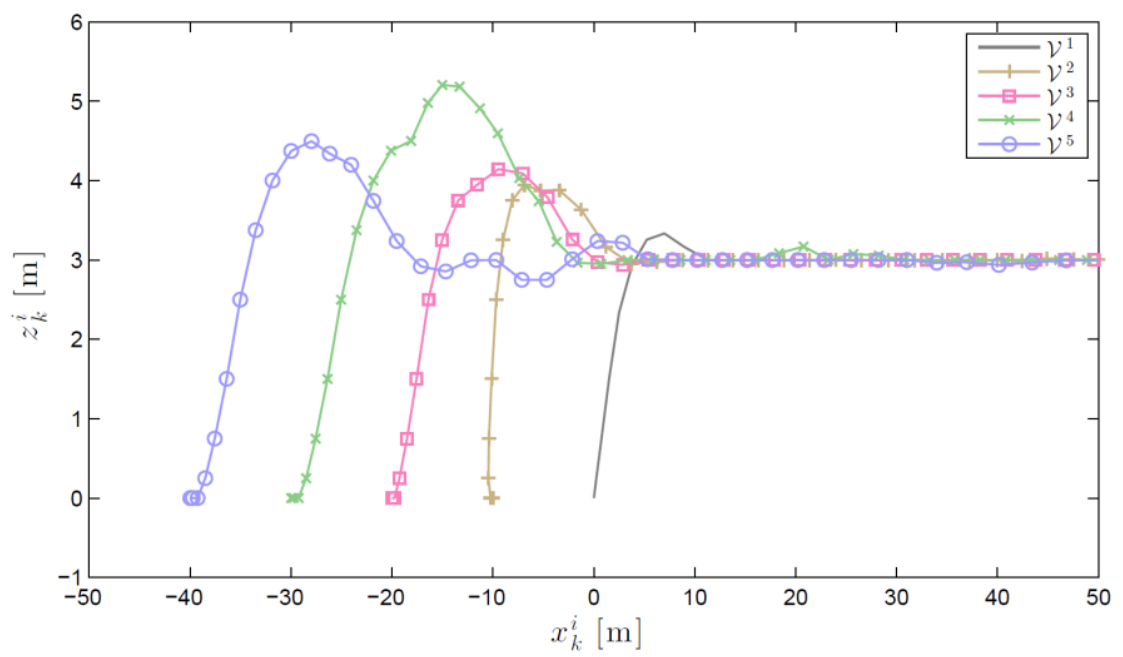

(b) Quadrotors trajectories projected into the X-Z plane

Figure 3. Trajectories followed by the five quadrotors in the horizontal plane (a) and in the vertical plane (b). Each vehicle is identified by a colour. The simulation is frozen at sample times $k=0, k=175$ and $k=350$
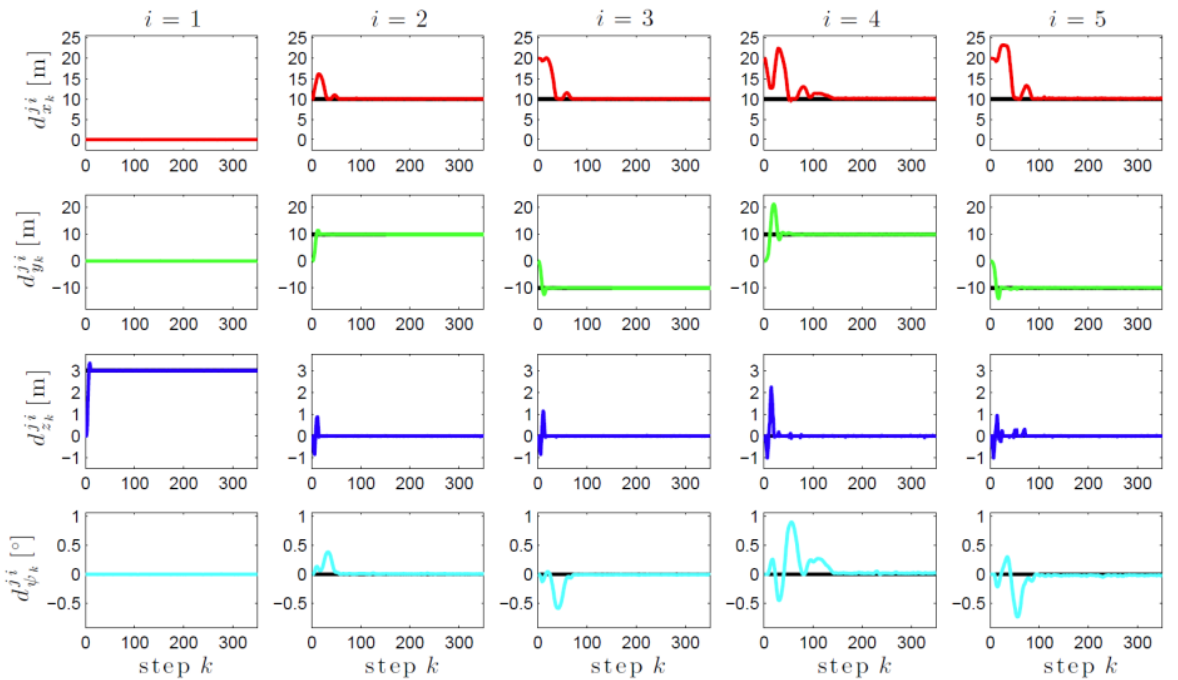

Figure 4. Components of the displacement vectors. The ideal values are plotted in black, while the real values are reported with different colours. The $i$-th column contains the relative distance between vehicle $i$ and vehicle $i-1$, read into the frame fixed to $\mathcal{V}^{i}$. The main leader distances (column 1) are referred instead to virtual leader $\mathcal{V}^{0}$

(i.e. point moving along the planar reference trajectory) 
The contribution of the proposed solution is that it permits to solve the formation control problem for a wide class of vehicles which satisfy a set of requirements typically possessed by unmanned vehicles. The proposed solution takes into account saturation constraints for the control actions, together with collision avoidance constraints. Moreover it is fully decentralized, thus each control agent must solve only a reduced optimization problem. Finally stability is granted by adding an auxiliary vector which imposes an upper bound to the system states. The authors are currently working to modify the proposed approach in order to deal with asynchronous communication and packet loss, by allowing a limited variable delay for each packet and introducing a fault tolerant policy whenever the delay exceeds the chosen thresholds. Both these aspects, together with a practical implementation of the proposed algorithm on a real system, are actually under investigation.

\section{References}

[1] B. Bethke, M. Valenti, J. How. UAV task assignment. IEEE Robotics \& Automation Magazine, 2008, Vol. 15, No. 1, 39-44.

[2] D. Lyon. A military perspective on small unmanned aerial vehicles. IEEE Instrumentation \& Measurement Magazine, 2004, Vol. 7, No. 3, 27-31.

[3] B. Mohr, D. Fitzpatrick. Micro air vehicle navigation system. IEEE Aerospace and Electronic Systems Magazine, 2008, Vol. 23, No. 4, 19-24.

[4] K. P. Valavanis. Advances in unmanned aerial vehicles: state of the art and the road to autonomy. Springer, The Netherlands, 2007.

[5] Y. Wang, P. Zhou, Q. Wang, D. Duan. Reliable Robust Sampled-Data $H_{\infty}$ Output Tracking Control with Application to Flight Control. Information Technology and Control, 2014, Vol. 43, No. 2, 175-182.

[6] M. Anderson, M. McKay, B. Richardson. Multi-robot automated indoor floor characterization team. In: Proceedings of the IEEE International Conference on Robotics and Automation, 1996, Vol. 2, pp. 1750-1753.

[7] W. Kang, H. Yeh. Co-ordinated attitude control of multi-satellite systems. International Journal of Robust and Nonlinear Control, 2002, Vol. 12, No. 2-3, 185-205.

[8] W. Dickson, R. H. Cannon, S. Rock. Symbolic dynamic modelling and analysis of object/robot-team systems with experiments. In: Proceedings of the IEEE International Conference on Robotics and Automation, 1996, Vol. 2, pp. 1413-1420.

[9] T. Vidal, M. Ghallab, R. Alami. Incremental mission allocation to a large team of robots. In: Proceedings of the IEEE International Conference on Robotics and Automation (ICRA), Minneapolis, Minnesota, USA, 1996, Vol. 2, pp. 1620-1625.

[10] X. Kang, H. Xu, X. Feng, Y. Tian. Formation evaluation of multi-AUV system for deep-sea hydrothermal plume exploration. In: Proceedings of the International Conference on Intelligent Human-Machine Systems and Cybernetics (IHMSC), 2009, Vol. 1, pp. 256-261.

[11] T. Balch, R. Arkin. Behavior-based formation control for multirobot teams. IEEE Transactions on Robotics and Automation, 1998, Vol. 14, No. 6, 926-939.
[12] J. Fredslund, M. Mataric. A general algorithm for robot formations using local sensing and minimal communication. IEEE Transactions on Robotics and Automation, 2002, Vol. 18, No. 5, 837-846.

[13] J. Lawton, R. Beard, B. Young. A decentralized approach to formation maneuvers. IEEE Transactions on Robotics and Automation, 2003, Vol. 19, No. 6, 933-941.

[14] D. Gu. A differential game approach to formation control. IEEE Transactions on Control Systems Technology, 2008, Vol. 16, No. 1, 85-93.

[15] P. Ogren, M. Egerstedt, X. Hu. A control Lyapunov function approach to multiagent coordination. IEEE Transactions on Robotics and Automation, 2002, Vol. 18, No. 5, 847-851.

[16] W. Ren, R. Beard. Formation feedback control for multiple spacecraft via virtual structures. IEE Proceedings on Control Theory and Applications, 2004, Vol. 151, No. 3, 357-368.

[17] K. H. Tan, M. Lewis. Virtual structures for highprecision cooperative mobile robotic control. In: Proceedings of the IEEE/RSJ International Conference on Intelligent Robots and Systems (IROS), Osaka, Japan, 1996, Vol. 1, pp. 132-139.

[18] K. Choi, S. Yoo, J. Park, Y. Choi. Adaptive formation control in absence of leader's velocity information. IET Control Theory \& Applications, 2010, Vol. 4, No. 4, 521-528.

[19] M. Defoort, T. Floquet, A. Kokosy, W. Perruquetti. Sliding-mode formation control for cooperative autonomous mobile robots. IEEE Transactions on Industrial Electronics, 2008, Vol. 55, No. 11, 3944-3953.

[20] J. Shao, G. Xie, L. Wang. Leader-following formation control of multiple mobile vehicles. IET Control Theory \& Applications, 2007, Vol. 1, No. 2, 545-552.

[21] S. Sheikholeslam, C. Desoer. Control of interconnected nonlinear dynamical systems: the platoon problem. IEEE Transactions on Automatic Control, 1992, Vol. 37, No. 6, 806-810.

[22] T. Dierks, S. Jagannathan. Neural network output feedback control of robot formations. IEEE Transactions on Systems, Man, and Cybernetics, Part B, 2010, Vol. 40, No. 2, 383-399.

[23] T. Sun, Y. Pan. Leader-based Consensus of Heterogeneous Nonlinear Multi-agent Systems. Mathematical Problems in Engineering, 2014, Article ID 519524, $1-6$.

[24] W. B. Dunbar, D. S. Caveney. Distributed Receding Horizon Control of Vehicle Platoons: Stability and String Stability. IEEE Transactions on Automatic Control, 2012, Vol. 57, No. 3, 620-633.

[25] W. B. Dunbar, R. M. Murray. Distributed receding horizon control for multi-vehicle formation stabilization. Automatica, 2006, Vol. 42, No. 4, 549-558.

[26] A. Fatehi, B. Sadeghpour, B. Labibi. Nonlinear System Identification in Frequent and Infrequent Operating Points for Nonlinear Model Predictive Control. Information Technology and Control, 2013, Vol. 42, No. 1, 67-76.

[27] A. Freddi, S. Longhi, A. Monteriù. Nonlinear Decentralized Model Predictive Control Strategy for a Formation of Unmanned Aerial Vehicles. In: Proceedings of the 2nd IFAC Workshop on Multi Vehicle Systems (MVS), Espoo, Finland, 2012, pp. 49-54.

[28] T. Keviczky, F. Borrelli, G. Balas. Decentralized receding horizon control for large scale dynamically 
decoupled systems. Automatica, 2006, Vol. 42, No. 12, 2105-2115.

[29] H. Fukushima, K. Kon, F. Matsuno. Model Predictive Formation Control Using Branch-and-Bound Compatible with Collision Avoidance Problems. IEEE Transactions on Robotics, 2013, Vol. 29, No. 5, 1308-1317.

[30] A. Guillet, R. Lenain, B. Thuilot, P. Martinet. Adaptable Robot Formation Control: Adaptive and Predictive Formation Control of Autonomous Vehicles. IEEE Robotics \& Automation Magazine, 2013, Vol. 21, No. 1, 28-39.

[31] H. Lim, Y. Kang, J. Kim, C. Kim. Formation control of leader following unmanned ground vehicles using nonlinear model predictive control. In: Proceedings of the IEEE/ASME International Conference on Advanced Intelligent Mechatronics, Singapore, 2009, pp. 945-950.

[32] T. P. Nascimento, A. P. Moreira, A. G. Scolari Conceicao. Multi-robot nonlinear model predictive formation control: Moving target and target absence. Robotics and Autonomous Systems, 2013, Vol. 61, No. 12, 1502-1515.

[33] T. Trindade Ribeiro, R. Ferrari, J. Santos, A. G. S. Conceicao. Formation control of mobile robots using decentralized nonlinear model predictive control. In: Proceedings of the IEEE/ASME International Conference on Advanced Intelligent Mechatronics, Wollongong, NSW, 2013, pp. 32-37.

[34] C. Zhang, T. Sun, Y. Pan. Neural Network ObserverBased Finite-Time Formation Control of Mobile Robots. Mathematical Problems in Engineering, 2014, Article ID 267307, 1-9.

[35] Y. H. Chang, C. W. Chang, C. L. Chen, C. W. Tao. Fuzzy sliding-mode formation control for multirobot systems: Design and implementation. IEEE Transactions on Systems, Man, and Cybernetics, Part B, 2012, Vol. 42, No. 2, 444-457.

L. Fang, P. Antsaklis. Decentralized formation tracking of multi-vehicle systems with nonlinear dynamics.
In: Proceedings of the 14th Mediterranean Conference on Control and Automation (MED), 2006, pp. 1-6.

[36] A. Fonti, A. Freddi, S. Longhi, A. Monteriù. Cooperative and decentralized navigation of autonomous underwater gliders using predictive control. In: Proceedings of the 18th IFAC World Congress, Milan, Italy, 2011, Vol. 18, No. 1, pp. 12813-12818.

[37] M. Vaccarini, S. Longhi. Networked decentralized MPC for formation control of underwater glider fleets. In: Proceedings of the 7th IFAC Conference on Control Applications in Marine Systems, Bol, Croatia, 2007, Vol. 7, pp. 63-68.

[38] M. Vaccarini, S. Longhi. Networked decentralized MPC for unicycle vehicles formation. In: Proceeding of the 7th IFAC Conference on Nonlinear Control Systems, Pretoria, South Africa, 2007, Vol. 7, pp. 603-608.

[39] T. Fossen. Handbook of Marine Craft Hydrodynamics and Motion Control. John Wiley \& Sons Ltd, 2011.

[40] P. Castillo, R. Lozano, A. Dzul. Modelling and control of mini-flying machines. Springer-Verlag New York Inc., 2005.

[41] A. Freddi, S. Longhi, A. Monteriù. A coordination architecture for UUV fleets. Intelligent Service Robotics, 2012, Vol. 5, No. 2, 133-146.

[42] J. Tamimi, P. Li. A new optimal control formulation to ensure the stability of NMPC systems. In: Proceedings of the 18th IFAC World Congress, Milan, Italy, 2011, Vol. 18, pp. 5495-5550.

[43] J. Nocedal, S. Wright. Numerical optimization. Springer Series in Operations Research and Financial Engineering, 2006, 2nd edition.

[44] H. Huang, G. Ma, Y. Zhuang, Y. Lv. Optimal Spacecraft Formation Reconfiguration with Collision Avoidance Using Particle Swarm Optimization. Information Technology and Control, 2012, Vol. 41, No. 2, 143-150.

[45] S. Bouabdallah, M. Becker, R. Siegwart. Autonomous miniature flying robots: coming soon! - research, development, and results. IEEE Robotics \& Automation Magazine, 2007, Vol. 14, No. 3, 88-98.

Received May 2014. 